\title{
INVESTIGACIÓN
}

Recibido: 15/02/2016 --- Aceptado: 19/05/2016 --- Publicado: 15/03/2017

\section{DISEÑO Y VALIDACIÓN DE UN CUESTIONARIO PARA CONOCER LA PRAXIS DOCENTE DEL PROFESORADO DE FLAUTA TRAVESERA EN CENTROS SUPERIORES DE LA COMUNIDAD VALENCIANA}

Ana María Botella Nicolás ${ }^{\mathbf{1}}$ : Universitat de València. España.

ana.maria.botella@uv.es

Guillem Escorihuela Carbonell: Conservatorio Superior de Música de Castellón. España.

escori@hotmail.com

\section{RESUMEN}

El objetivo del presente estudio es diseñar y validar un cuestionario para conocer la opinión del profesorado de las enseñanzas superiores de flauta travesera de la Comunidad Valenciana sobre la praxis docente. La población de referencia está formada por el conjunto de profesores de flauta travesera de los tres conservatorios superiores de música de la Comunidad Valenciana: CSM "Oscar Esplà" de Alicante, CSM "Joaquín Rodrigo" de Valencia y CSM "Salvador Seguí" de Castellón. El cuestionario fue enviado a toda la población y la muestra generadora de datos resultó ser del $100 \%$. Sin embargo dos de los encuestados no respondieron, por tanto tenemos un sesgo del 33'33\%. Se considera que el estudio sigue siendo viable debido a que hay por lo menos 1 sujeto que responde de cada centro, por ello hay información de los tres centros de la Comunidad Valenciana. Los resultados obtenidos muestran que el cuestionario es válido y fiable para conocer la opinión sobre la praxis docente de la flauta en los conservatorios superiores de la Comunidad Valenciana.

PALABRAS ClAVE: Cuestionario - Enseñanzas superiores - Conservatorio Validación - Profesorado - Flauta - Comunidad Valenciana.

\section{DESIGN AND VALIDATION OF A QUESTIONNAIRE TO DETERMINE TEACHING PRAXIS FLUTE TEACHER IN HIGHER CENTERS OF VALENCIAN COMMUNITY}

\footnotetext{
1 Ana María Botella Nicolás: profesora contratada doctora del Departamento de Didáctica de la Expresión musical, plástica y corporal de la Facultad de Magisterio de la Universitat de València. Correo: ana.maria.botella@uv.es
} 
Botella Nicolás, A. M.; Escorihuela Carbonell, G. Diseño y validación de un cuestionario para conocer la praxis docente del profesorado de flauta travesera en centros superiores de la comunidad valenciana

\section{ABSTRACT}

The aim of this study is to design and validate a questionnaire to determine what is the opinion of teachers of the higher teachings of flute of Valencia on teaching practice. The reference population is formed by all teachers of flute of the top three music conservatories of Valencian Community: CSM "Oscar Esplá" of Alicante, CSM "Joaquín Rodrigo" of Valencia and CSM "Salvador Segui" of Castellón. The questionnaire was sent to all the population and generating data sample was found to be $100 \%$. However two of the respondents did not respond, so we have a bias of $33^{\prime} 33 \%$. It is considered that the study is still viable because there is at least one subject responds each centre, so we have information from the three centres of Valencian Community. The results show that the questionnaire is valid and reliable for getting feedback on teaching practice of the flute in the conservatories of Valencian Community.

KEY WORDS: Questionnaire - Higher education teaching - Conservatory Validation - Teachers - Flute - Valencian Community.

\section{DESENHO E VALIDAÇÃO DE UM QUESTIONÁRIO PARA CONHECER A PRAXIS DOCENTE DO PROFESSORADO DE FLAUTA TRANSVERSAL NOS CENTROS SUPERIORES DA COMUNIDADE VALENCIANA}

\section{RESUMO}

O objetivo do presente estudo e desenhar e validar um questionário para conhecer a opinião do professorado do ensino superior de flauta transversal da Comunidade Valenciana sobre a práxis docente. A população de referência esta formada por um conjunto de professores de flauta transversal dos três conservatórios superiores de música da Comunidade Valenciana: CSM "Oscar Esplá" de Alicante, CSM "Joaquin Rodrigo" de Valência e CSM "Salvador Seguí" de Castellón. O questionário foi enviado a toda população e a mostra geradora de dados resultou ser de $100 \%$. Mesmo assim dois dos entrevistados não responderam, portanto temos uma inclinação de um $33,33 \%$. Considera-se que o estudo segue sendo viável devido ter pelo menos um sujeito que responde de cada centro, por isso tem informação dos três centros da Comunidade Valenciana. Os resultados obtidos mostram que o questionário é valido e confiável para conhecer a opinião sobre a práxis docente da flauta nos conservatórios superiores da Comunidade Valenciana.

PALAVRAS CHAVE: Questionário - Ensinos superiores - Conservatório - Validação - Professorado - Flauta - Comunidade Valenciana 


\section{Cómo citar el artículo}

Botella Nicolás, A. M.; Escorihuela Carbonell, G. (2017). Diseño y validación de un cuestionario para conocer la praxis docente del profesorado de flauta travesera en centros superiores de la comunidad valenciana. [Designing and validating a questionnaire to determine the teaching praxis of the transverse flute professors in higher education centers of Valencian community]. Revista de Comunicación de la SEECI, 43, 01-13. doi: http://dx.doi.org/10.15198/seeci.2017.42.01-13 Recuperado de http://www.seeci.net/revista/index.php/seeci/article/view/392

\section{INTRODUCCIÓN}

La praxis docente no es inmanente en el tiempo, se trata de un devenir cambiante y sujeto a multitud de variables. Esto hace difícil su revisión, que muchas veces se ve relegada a un estudio de la metodología pero no de la acción docente (Botella y Escorihuela, 2014).

La investigación sobre la didáctica de los instrumentos y su praxis docente se ha abordado desde diferentes campos, tanto a nivel de enseñanzas profesionales como amateurs o complementarias. La de la flauta dulce o de pico es la que más ha sido estudiada, ya que resulta una herramienta docente importante en la asignatura de música en la Educación Primaria y la Secundaria. Sin embargo, apenas existen trabajos científicos relacionados con los instrumentos que se estudian de manera profesional en los Conservatorios. Son los instrumentos de cuerda los que más han sido investigados en este campo. Esto se debe a su gran variedad técnica y metodológica, muy arraigada a las escuelas nacionales y que admite una fácil diferenciación y medición de sus resultados. En la mayoría de casos, los docentes tienen claros los objetivos que se pretenden alcanzar y los contenidos a desarrollar, pero muchas veces se deja de lado la reflexión en torno a la práctica en el aula. Es decir, se conocen bien los conceptos técnicos pero no se hace una reflexión sobre cómo transmitir los conocimientos al alumno (Botella y Martínez, 2015).

La didáctica instrumental de la flauta en los ciclos superiores está poco tratada. Se sabe poco de lo que el profesorado enseña y cómo lo enseña, de las corrientes flautísticas que se están siguiendo y del tipo de experto de la flauta que una vez acabada su educación superior está saliendo al mercado musical. En definitiva, qué "producto" musical está lanzando al mundo profesional el sistema educativo. Por tanto, como dicen Botella y Escorihuela (2016, p. 412): "se necesita saber qué y cómo se está enseñando para saber en qué y cómo mejorar esta praxis y revisar, incluso exportar, las características propias del tipo de enseñanza flautística que se da".

Desde que en 1707 se publicara el considerado primer método de flauta, Principios de la flauta traversa o flauta de Alemania de Jacques-Martin Hotteterre, se han sucedido los métodos y libros de estudio de este instrumento. Durante el siglo XIX la enseñanza instrumental evolucionó con las mejoras en la construcción de los 
instrumentos. Así se propició la aparición de métodos, algunos autodidactas, impulsando la profesionalización del músico y despopularizando la música que se tocaba en los hogares. La sistematización que debe imponerse en todo método puede verse en el Célebre Método de $\mathrm{H}$. Altès, uno de los clásicos junto con los de Devienne, Tulou, Hugot-Wunderlich o Taffanel. El Romanticismo, como movimiento estético, desembocará en un impulso definitivo a la mecanización de la flauta travesera, desarrollada y popularizada por Theobald Boehm. La adopción de la flauta Boehm o no por parte de los profesores y los flautistas destacados del momento, marca un antes y un después en la enseñanza de este instrumento, pudiendo catalogar los métodos de flauta en antiguos o modernos en función de para qué sistema de flauta están escritos. Esta catalogación dicotómica no es excluyente en el sentido de que existen métodos que son válidos para más de un sistema o modelo de flauta, pero ayuda a comprender la aparición de abundante literatura entorno al aprendizaje de la nueva flauta que ha llegado hasta hoy en día.

Además del método o métodos, la enseñanza de la flauta travesera también puede verse influenciada por lo que se ha venido a denominar escuela. Este concepto abarca la metodología empleada por el maestro o profesor, o el conjunto de docentes de una institución. En el ámbito del arte la escuela define al grupo formado por los seguidores, admiradores o aprendices de un individuo que actúa como maestro o fuente de inspiración. Las escuelas nacionales de flauta travesera han jugado un papel importante en la generalización y el exponencial de las técnicas y los métodos que han conseguido ser atemporales y de probada solvencia. Se pueden diferenciar dos grandes escuelas flautísticas: la francesa y la inglesa. No obstante, a partir del siglo XX emerge con fuerza la escuela americana, y tampoco hay que dejar de lado la influencia que en su día tuvo la escuela centroeuropea (Alemania y Austria).

Con todo ello, se tiene que tener en cuenta una serie de variables a la hora de investigar la praxis docente. Éstas no siempre son estables, pues en cada país se toca la flauta con unas características propias, normalmente resultado de la influencia de uno o más conocidos instrumentistas del lugar en cuestión. En el caso de la escuela, los viajes, las grabaciones y la radio están borrando poco a poco las diferencias. La escuela como concepto de ejecución basado en la enorme influencia de un gran flautista a través de su método pedagógico, sus libros y su manera de tocar queda en entredicho (Wye, 1988).

Esta investigación refleja la práctica docente en esta etapa y aborda otras cuestiones que hacen referencia al objetivo general que los docentes pretenden conseguir con su alumnado y a la metodología que utilizan en sus clases de flauta travesera.

\section{OBJETIVOS}

El objetivo principal de este estudio fue conocer la praxis docente de los profesores de las enseñanzas superiores de flauta travesera de la Comunidad Valenciana. Este objetivo marco que engloba toda la investigación es el que permitirá ir deshilando el entramado que supone la didáctica del instrumento en este campo de estudio. Para 
Botella Nicolás, A. M.; Escorihuela Carbonell, G. Diseño y validación de un cuestionario para conocer la praxis docente del profesorado de flauta travesera en centros superiores de la comunidad valenciana

llevar a cabo este cometido se plantean los siguientes objetivos específicos que se relacionan directamente con la estructura de la investigación:

1. Describir la práctica didáctica de los Conservatorios Superiores de la C. Valenciana.

2. Observar y determinar a qué perfil flautístico se adscribe cada profesor y cómo influye en el tipo de alumnado.

3. Conocer los autores de referencia que se interpretan tanto en el repertorio como en los libros de técnica y de estudios, y si los profesores siguen un programa común de centro o cada uno prepara su programación.

4. Descubrir en qué grado el bagaje musical de cada profesor influye en su manera de impartir clase de flauta y de programar.

\section{METODOLOGÍA}

Se emplean los siguientes procedimientos metodológicos para alcanzar los objetivos propuestos:

1. Una revisión bibliográfica y un estudio histórico-musical que se hace en base a un detallado análisis de fuentes primarias y secundarias sobre lo que hasta el momento se ha escrito del estudio de la flauta. Haciendo referencia tanto a tratados del siglo XVII y XVIII, como a los escritos más actuales. Destacan los hallazgos sobre la flauta en España durante el siglo XIX.

2. Un estudio vinculado a la praxis de la flauta en las aulas de los centros superiores de música de la Comunidad Valenciana. Sirve para conocer la actitud del profesorado, mediante un cuestionario que les permita expresar su parecer acerca de la mejor manera de enseñar este instrumento y cuáles son los pilares en los que fundamentan la formación que dan a los alumnos. Un cuestionario mediante el cual los profesores podrán aportar información sobre sus prácticas docentes.

\subsection{Poblaciones y muestras.}

Las técnicas de muestreo que se utilizan en investigación permiten determinar la parte de la realidad estudiada (población) que debe examinarse con objeto de hacer inferencias sobre dicha población. Se trata de obtener una muestra adecuada que permita lograr una versión simplificada de la población y que reproduzca sus rasgos básicos con la precisión fijada. La población de referencia de esta investigación está constituida por el conjunto de profesores de flauta travesera de los conservatorios superiores de música de la Comunidad Valenciana. La siguiente fórmula nos permite calcular el tamaño de la muestra para poblaciones finitas:

$$
\text { En ella: } \quad n=Z_{\alpha}^{2} \frac{N \cdot p \cdot q}{i^{2}(N-1)+Z_{\alpha}^{2} \cdot p \cdot q}
$$


- La $\mathrm{n}$ es el tamaño que necesita tener la muestra, conociendo el tamaño de la población $\mathrm{N}$. $\mathrm{N}=6$ en el caso de los profesores de flauta travesera de los conservatorios superiores de la Comunidad Valenciana.

- La Z es el valor correspondiente a la distribución de Gauss 1,96 para $\alpha=0,05$ si trabajamos con un nivel de confianza (1- $\alpha$ ) del $95 \%$. El nivel de confianza es la probabilidad de que la estimación efectuada se ajuste a la realidad. Es la probabilidad de que el intervalo construido en torno al estadístico capte el verdadero valor del parámetro.

- La p es la variabilidad del parámetro a estimar que generalmente se obtiene de estudios previos o piloto, pero que cuando se desconoce, como en este caso, se aplica la opción más desfavorable posible $(p=0,5)$, que hace mayor el tamaño muestral. La $q=1-p$, siendo también en este caso $q=0,5$.

- La i es el error que está previsto cometer y nos indica la amplitud del intervalo de confianza entre muestra y muestra. Este error mide la variabilidad de muestras diferentes obtenidas a partir de la misma población.

La población de referencia de esta investigación está constituida por: el conjunto de profesores de flauta travesera de los tres conservatorios superiores de música de la Comunidad Valenciana, estos son: CSM "Oscar Esplà" de Alicante, CSM "Joaquín Rodrigo" de Valencia y CSM "Salvador Seguí" de Castellón. Según los sitios web de estos centros, el Instituto Superior de Enseñanzas Artísticas de la Comunidad Valenciana (ISEACV) y la propia Conselleria d'Educació, titular de los tres, constan seis profesores del instrumento flauta travesera, a razón de dos profesores por centro. Pertenecen al cuerpo de personal docente de Conselleria, entre los que pueden ser catedráticos, profesores en comisión de servicio habilitados como catedráticos o interinos. Los datos están tomados referentes al curso 2012/2013. Trabajamos con un nivel de confianza del $95 \%$ y un margen de error del $5 \%$. Por lo tanto, los resultados de esta investigación se derivan de muestras muy significativas y representativas de la población.

\subsection{El cuestionario. Construcción y validación}

El instrumento de recogida de la información fue el cuestionario con el título "Cuestionario para conocer la Praxis Docente del Profesorado de Flauta Travesera en Centros Superiores de la Comunidad Valenciana". Los criterios básicos que se han tenido en cuenta en su elaboración han sido la sencillez, precisión y concreción de los ítems que los componen y la discreción y preservación del anonimato en el manejo de la información recabada. Nos decantamos por este procedimiento de recogida de información altamente estructurado, en el que se plantean una serie de preguntas en torno a las variables que se quieren medir. Con ello conseguimos reunir mucha información en relativamente poco tiempo.

En primer lugar se diseñó el cuestionario a partir de la revisión de la bibliografía existente. La primera versión fue revisada por profesionales expertos del ámbito universitario especializados en evaluación de programas, que fue modificada después, teniendo en cuenta sus aportaciones y sugerencias. De este modo, se han revisado los aspectos necesarios, matizando la redacción y expresión de las preguntas, cuidando la 
utilización de un vocabulario más adaptado a los profesores y redistribuyendo el orden de los ítems. Queremos reseñar que esta revisión preliminar ha sido básica y fundamental en la elaboración del cuestionario. La validez de contenido es el grado en que una prueba representa de forma adecuada lo que se ha realizado (Thomas y Nelson, 2007; Ortega, Jiménez, Palao y Sainz, 2008, citados en Garrido, Zagalaz, Luque y Granados, 2010). Una segunda revisión fue llevada a cabo por contraste de expertos de validación de contenido que han verificado que los ítems propuestos miden la didáctica instrumental de la flauta. Se trata de tres profesionales ${ }^{2}$ en la materia, siguiendo así el criterio de la triangulación. Éstos presentan una dilatada carrera en la enseñanza y la práctica instrumental de la flauta, tanto en conservatorios y orquestas:

- Magdalena Martínez, solista de la Orquestra Simfònica de Barcelona y Nacional de Catalunya (OBC) y desde 2006 flauta solista de la Orquestra de la Comunitat Valenciana del Palau de les Arts, ha sido profesora de la Universidad de Alcalá de Henares y de l'Escola Superior de Música de Catalunya, además de impartir diversas masterclasses y postgrados.

- Miguel Llopis, en el campo de la música sinfónica ha colaborado con la Orquesta de Valencia, Orquesta Sinfónica del Mediterráneo, Sinfónica de Valencia, Orquesta Sinfónica de Madrid y desarrolla su actividad docente en el Conservatorio Profesional de Música de Torrent.

- Federico Peris, ha sido miembro de la Orquesta Ciudad de Valladolid y ha desarrollado su labor como profesor en el Conservatorio Superior de Zaragoza, Profesional de Música de Gijón, Profesional de Música Amaniel de Madrid, Profesional de Música de Ontinyent, Profesional de Valencia y actualmente en el Conservatorio Profesional de Música de Catarroja. Ha colaborando con la Orquesta Sinfónica de Madrid y Orquesta de RTVE. Los dos últimos son miembros del Seminario Valenciano de la Flauta, firmando con otros de sus compañeros publicaciones sobre la didáctica del instrumento.

Por tanto, en la elaboración del cuestionario se han tenido en cuenta:

- Definición del constructo a medir.

- Propósito de la escala.

- Composición de los ítems (número, contenido, definición y ordenación).

- Prevención de los sesgos en su cumplimentación y codificación de las respuestas.

Las variables de información y clasificación de este estudio son las que permiten la estructuración del cuestionario configurando los distintos ítems. Éstas son: edad, sexo, nacionalidad, centro de trabajo, cargo directivo y número de alumnos.

Por lo que se refiere a su estructura, el cuestionario se compone de un cuadro inicial que contiene las variables de clasificación que permiten una primera caracterización de la muestra. Les siguen 5 dimensiones relativas al perfil del profesor, colocación y

\footnotetext{
${ }^{2}$ Magdalena Martínez, Miguel Llopis y Federico Peris.
} 
Botella Nicolás, A. M.; Escorihuela Carbonell, G. Diseño y validación de un cuestionario para conocer la praxis docente del profesorado de flauta travesera en centros superiores de la comunidad valenciana

embocadura, estudio del sonido, la digitación y la articulación, estudio y repertorio. Se trata de un total de 93 ítems, que recogen información relativa a diversas variables de estudio. Respecto a la tipología y caracterización de las preguntas, el cuestionario contiene preguntas cerradas, semiabiertas y abiertas, distribuidas en las siguientes dimensiones, tal y como aparece en la tabla 1:

Tabla 1: Cuestionario para conocer la Praxis Docente del Profesorado de Flauta Travesera en Centros Superiores de la Comunidad Valenciana

\begin{tabular}{|c|c|c|}
\hline DIMENSIONES & NOMBRE & $\underline{\text { ITTEMS }}$ \\
\hline $\begin{array}{l}\text { Variables de } \\
\text { clasificación }\end{array}$ & $\begin{array}{l}\text { Sexo, edad, nacionalidad, centro de trabajo, } \\
\text { cargo directivo y número de alumnos. }\end{array}$ & $1-6$ \\
\hline Perfil del profesor & $\begin{array}{l}\text { Años de docencia, cátedra, estudios, principales } \\
\text { profesores, estudios en el extranjero, horas de } \\
\text { estudio diario, trabajo en orquesta, música de } \\
\text { cámara, actividad solista, estilo musical } \\
\text { preferido, escuela nacional de flauta, flautista } \\
\text { de referencia, dedicación a la investigación, } \\
\text { otros estudios superiores, cursos y seminarios, } \\
\text { aportaciones a revistas y objetivo principal para } \\
\text { con sus alumnos. }\end{array}$ & $7-37$ \\
\hline $\begin{array}{l}\text { Colocación y } \\
\text { embocadura }\end{array}$ & $\begin{array}{l}\text { Importancia a la colocación, tiempo dedicado en } \\
\text { clase, referencia a órganos y músculos del } \\
\text { proceso respiratorio, tipo de ejercicios, libros de } \\
\text { ejercicios, importancia a la posición corporal, } \\
\text { importancia a la respiración, ejercicios propios y } \\
\text { mecanización o no de los ejercicios. }\end{array}$ & $38-50$ \\
\hline $\begin{array}{l}\text { Estudio del } \\
\text { sonido, la } \\
\text { digitación y la } \\
\text { articulación }\end{array}$ & $\begin{array}{l}\text { Importancia del sonido, tiempo dedicado, } \\
\text { intención en el trabajo con el alumno, tipo de } \\
\text { ejercicios, libros, material propio, vibrato, } \\
\text { cambios de color, posibilidades tímbricas, trabajo } \\
\text { de la digitación y articulación, tipo de ejercicios, } \\
\text { libros de técnica. }\end{array}$ & $51-71$ \\
\hline Estudios & $\begin{array}{l}\text { Libros de estudios, preferencia de libros, estilos } \\
\text { y periodos, estudios tradicionales y } \\
\text { contemporáneos. }\end{array}$ & $72-77$ \\
\hline
\end{tabular}




\begin{tabular}{|c|c|c|}
\hline $\begin{array}{c}\text { Repertorio } \\
\text { orquestal y obras } \\
\text { del repertorio }\end{array}$ & $\begin{array}{l}\text { Frecuencia de la asignatura de repertorio, } \\
\text { imparte o no la asignatura, libros de solos } \\
\text { orquestales, perfil del profesor de repertorio, } \\
\text { prácticas orquestales, instrumentos de la familia } \\
\text { de la flauta, obras del repertorio que se } \\
\text { trabajan, consenso entre profesores y profesor- } \\
\text { alumno, estilos que se trabajan, tipo de obra } \\
\text { más interpretada, preparación para tocar en } \\
\text { público y observaciones. }\end{array}$ & 78-93 \\
\hline & $\begin{array}{l}\text { TOTAL } \\
\text { Tu3 }\end{array}$ & $\mathbf{9 3}$ \\
\hline
\end{tabular}

Las preguntas cerradas generan distintas posibilidades de respuestas: 1) excluyentes (respuestas dicotómicas afirmativas o negativas, de grado de importancia); y 2) con varias opciones de respuesta en las que se puede señalar más de una alternativa. Optamos por las preguntas dicotómicas porque requieren de un menor esfuerzo por parte de los encuestados, son sencillas de contestar y mantienen atento al sujeto en el tema. Se utilizaron escalas de tipo Likert en muchos de sus ítems, pues pensamos que a través de ellas podíamos conseguir la reacción favorable o desfavorable, positiva o negativa, sin olvidarnos nunca que estamos midiendo sus actitudes ante este tipo de música.

Las preguntas semiabiertas se derivan directamente de las anteriores, cuando se contempla la posibilidad de añadir alternativas no incluidas en el cuestionario para enriquecer el análisis. Con las preguntas abiertas se pretende que puedan opinar libremente sobre lo que se les demanda e incluir la información que deseen sobre sus situaciones personales. Estas preguntas además les permiten matizar las respuestas cerradas y añadir nuevas perspectivas sobre el tema tratado.

Una vez diseñado el borrador definitivo, delimitada la información, formuladas las preguntas, definido el número de ellas y ordenadas, hay que realizar una evaluación de las propiedades métricas de la escala. El cuestionario se ha sometido a:

- Validez de contenido. Se refiere a si el cuestionario elaborado, y por tanto los ítems elegidos, son indicadores de lo que se pretende medir. Se trata de exponer el documento a la valoración de investigadores y expertos, que deben juzgar la capacidad de éste para evaluar todas las dimensiones que deseamos medir.

- Validez de constructo. Evalúa el grado en que el instrumento refleja la teoría del fenómeno o del concepto que mide. La validez de construcción garantiza que las medidas que resultan de las respuestas del cuestionario pueden ser consideradas y utilizadas como medición del fenómeno que se quiere medir. Puede ser calculada por diversos métodos, pero los más frecuentes son el análisis factorial y la matriz multirasgo-multimétodo. 
Botella Nicolás, A. M.; Escorihuela Carbonell, G. Diseño y validación de un cuestionario para conocer la praxis docente del profesorado de flauta travesera en centros superiores de la comunidad valenciana

- Validez de criterio. Relación de la puntuación de cada sujeto con un Gold Standard que tenga garantías de medir lo que se desea. No siempre hay indicadores de referencia, por lo que, muchas veces se recurre a utilizar instrumentos que han sido respaldados por otros estudios.

\section{DISCUSIÓN}

Después del análisis de los datos y resultados, del estudio se deriva que:

La mayoría de la muestra son mujeres, frente a un solo hombre. Al contrario de lo que sucede en el resto de países europeos, donde la movilidad del profesorado es una constante, en la Comunidad Valenciana todos los profesores son españoles. Por lo general, el número de alumnos varía bastante, aunque no se ha encontrado razón alguna en los análisis relacionales. Se puede fijar un promedio entre 14 y 15 alumnos por profesor.

Los profesores del Conservatorio de Valencia son los más mayores de edad y los que más antigüedad en el cuerpo presentan, dedicándose más tiempo a la docencia. Además son los dos únicos catedráticos de flauta travesera de la Comunidad. Así pues, es posible sostener que el profesorado de los conservatorios de Castellón y Alicante es más joven y tiene menos experiencia, mientras que Valencia se consolida como el centro que aglutina a los catedráticos con más experiencia.

La Comunidad Valenciana presenta una plantilla de profesores formados en los conservatorios de Valencia y Alicante, que compaginan la doble vertiente del músico: interpretativa y pedagógica. El 100\% realizó estudios en el extranjero (Francia, Alemania, Reino Unido e Italia). En sus currículums destacan los profesores Jesús Campos, Alain Marion y Peter Lukas Graf, ya que coinciden en la formación de algunos sujetos. Además, por lo general todos realizan actividades como concertistas, con recitales anuales. Se trata de un grupo de flautistas en activo, ya que continúan estudiando una media de dos horas diarias. Además tienen una gran experiencia en música de cámara. Esto entra en consonancia con el ideal de profesor de instrumento que expone Purser (2005), un docente activo y reconocido como buen instrumentista, a la vez que reflexivo y familiarizado con la filosofía del proceso de enseñanza-aprendizaje y que base la formación de conservatorio en algo más que una educación en la destreza instrumental.

Prácticamente la totalidad de la muestra se adhiere a aquello que afirma Wye (1988) de que hoy en día hay tantas escuelas como profesores influyentes. En cuanto al flautista de referencia, el 75\% coincide en la persona de Emmanuel Pahud.

El profesorado utiliza tanto libros como métodos provenientes de todas las escuelas, por tanto, se inspiran de todas las corrientes que aplican en función de cada caso en particular. Sin embargo, la forma de proceder en la praxis que indican sus respuestas en cuanto a los pilares del instrumentista: colocación, sonido, técnica de dedos y articulación, constituyen la base de la escuela francesa. 
Son muchos los nexos de unión en la praxis docente de estos centros. Los más destacados son los referentes a los ejercicios técnicos y de sonoridad, así como en cuanto a los libros de estudios que se programan. El abanico de métodos usados es muy amplio. Todos emplean material de diversas escuelas (francesa, italiana, alemana), sin embargo los títulos son coincidentes. Esto implica una praxis común que puede ser objeto de generalización. Así, se puede decir que apenas hay diferencias reseñables entre centros de la Comunidad Valenciana al respecto.

Tal y como sucede en la investigación de Purser (2005), se encuentran muchas similitudes. Éste descubrió a un grupo de artistas que se encontraban en distintas etapas de desarrollo en sus filosofías de enseñanza. Sin embargo, hubo un consenso sobre muchas cuestiones. Con todo ello, podemos hacer nuestra una de sus conclusiones: "es difícil no llegar a la conclusión de que algún foro para el intercambio de experiencias y modos de buenas prácticas docentes sería beneficioso" (p. 298).

\section{CONCLUSIONES}

La música es un arte que necesita esencialmente de la presencia de un mediador entre el creador y el público al que va destinado el producto artístico. Este mediador es el intérprete (Botella y Escorihuela, 2014). El propósito de este estudio ha sido diseñar y validad un cuestionario para conocer cómo es la praxis docente de los profesores de flauta travesera de los centros superiores de música de la Comunidad Valenciana. Todo ello, teniendo en cuenta que el futuro intérprete tendrá que aprender a leer correctamente la partitura, penetrando en el sentido de los escritos, para poder apreciar su valor estético. Al mismo tiempo se precisa que desarrolle la destreza necesaria en el manejo del instrumento para que la ejecución de ese texto musical adquiera su plena dimensión de mensaje expresamente significativo y pueda transmitir así, de manera convincente, la emoción del orden estético que en el espíritu del intérprete despierta la obra musical cifrada en la partitura.

Así, el cuestionario da validez al estudio y puede ser utilizado para medir actitudes relacionadas con la praxis docente del profesorado de flauta travesera de cetros superiores. Además puede servir de modelo para la investigación de cualquier enseñanza performativa musical, en tanto que su estructura se ajusta al prototipo de preparación que se da en los conservatorios.

Los resultados describen la praxis, tal y como pretendía el cuestionario, además permiten implementar la mejora de la didáctica de la flauta, en tanto que la comunidad docente puede tomar nuevas ideas y distintos puntos de vista a la hora de abordar los procesos educativos. Tanto las semejanzas como las diferencias encontradas enriquecen la docencia y presentan un modelo fiable y válido del paradigma de flautista profesional que se forma en la Comunidad Valenciana. 
Botella Nicolás, A. M.; Escorihuela Carbonell, G. Diseño y validación de un cuestionario para conocer la praxis docente del profesorado de flauta travesera en centros superiores de la comunidad valenciana

\section{REFERENCIAS}

Botella, A. M. y Escorihuela, G. (2014). Análisis de la praxis docente de los profesores de las enseñanzas superiores de flauta travesera de la Comunidad Valenciana. El Artista: revista de investigaciones en música y artes plásticas, 11, 65-87.

Botella, A. M. y Fuster, V. (2015). Estudio y análisis de los métodos de iniciación a la viola más utilizados por el profesorado de las enseñanzas elementales de la provincia de Valencia. Revista de Comunicación de la SEECI, 38, 146-164.

Botella, A. M. y Escorihuela, G. (2016). Educación performativa: La enseñanza superior de la flauta travesera en la Comunidad Valenciana. Actas del II Congreso Nacional de Conservatorios Superiores de Música, pp. 410-425.

Garrido, M. E., Zagalaz, M. L., Torres, G. y Romero, S. (2010). Diseño y validación de un cuestionario para técnicos deportivos acerca de su opinión sobre las actitudes de padres y madres en el deporte (CTPMD). Cuadernos de Psicología del Deporte, $10(2), 7-21$.

Ortega, E., Jiménez, J.M., Palao, J.M. y Sainz, P. (2008). Diseño y validación de un cuestionario para valorar las preferencias y satisfacciones en jóvenes jugadoras de baloncesto. Cuadernos de Psicología del Deporte, 8(2), 39-58.

Thomas, J. R. y Nelson, J. K. (2007). Métodos de investigación en actividad física. Barcelona: Paidotribo

Purser, D. (2005). Performers as teachers: exploring the teaching approaches of instrumental teachers in conservatoires. British Journal of Music Education, 22, 287-298. doi:10.1017/S0265051705006546

Wye, T. (1988). La flauta como es debido. Madrid: Mundimúsica Garijo.

\section{AUTORES \\ Ana María Botella Nicolás}

Doctora en pedagogía por la Universitat de València. Es Licenciada en Geografía e Historia, especialidad Musicología y maestra en Educación Musical, por la Universidad de Oviedo. Grado profesional en la especialidad de piano. Durante el año 2001 obtiene por oposición plaza en el cuerpo de profesores de música de enseñanza secundaria en Alicante (actualmente en excedencia). Ha presentado diversas comunicaciones en jornadas y congresos sobre didáctica de la música así como distintas publicaciones. Es profesora contratada doctora del departamento de didáctica de la Expresión Musical, Plástica y Corporal de la Facultad de Magisterio de la Universitat de València. Forma parte de la Comisión de Coordinación Académica del Master Universitario en Profesor/a de enseñanza secundaria de la UVEG y del Máster de Investigación en didácticas específicas. Desde Febrero de 2015 dirige el a 
Botella Nicolás, A. M.; Escorihuela Carbonell, G. Diseño y validación de un cuestionario para conocer la praxis docente del profesorado de flauta travesera en centros superiores de la comunidad valenciana

Aula de música del Vicerrectorado de Cultura e Igualdad.

http://orcid.org/0000-0001-5324-7152

ResearchID: N-1375-2014

\section{Guillem Escorihuela Carbonell}

Titulado Superior en Música en la especialidad de Flauta Travesera por el Conservatorio Superior de València. Máster en Profesor de Educación Secundaria y Máster de Investigación en Didácticas Específicas por la Universitat de València. Máster Universitario en Interpretación Musical por la Universidad Internacional de Andalucía. Profesor del Departamento de Música de Cámara del Conservatorio Superior de Música de Castellón. Doctorando en Didáctica de la Música, donde investiga sobre la enseñanza de la flauta travesera.

http://orcid.org/0000-0001-5735-3841 